\title{
JANUS KINASE V617F MUTATION DETECTION IN PATIENTS WITH MYELOFIBROSIS
}

\author{
Nikolova $\mathrm{D}^{1,2, *}$, Yordanov $\mathrm{A}^{2}$, Damyanova $\mathrm{V}^{1,2}$, Radinov $\mathrm{A}^{2}$, Toncheva $\mathrm{D}^{1}$ \\ *Corresponding Author: Dragomira Nikolova, Ph.D., Department of Medical Genetics, Medical Fac- \\ ulty, Medical University Sofia, 2 Zdrave Street, 1431 Sofia, Bulgaria. Tel: +359888-103-456, \\ E-mail: dmb@abv.bg
}

\begin{abstract}
Myelofibrosis (MF) is characterized by a presence of an extra fibrous tissue in the bone marrow and additional hematopoiesis. The somatic mutation in the Janus kinase 2 (JAK2) gene (V617F) occurs gradually and is detected in about $50.0 \%$ of myelofibrosis or essential thrombo-cytopenia (ET) patients. Our aim was to determine the genotype status according to the carriers of the V617F mutation in patients with MF at the Hematology Ward of the University Hospital "Ivan Rilski" in Sofia, Bulgaria. DNA samples were isolated from venous blood of patients with various hematological disorders. DNA was amplified by polymerase chain reaction (PCR) and subsequent restriction analysis was performed using a $B s a X I$ restriction enzyme. The genotype status was determined on $2.0 \%$ agarose gel. We analyzed 38 patients initially suspected of carrying MF or osteomyelofibrosis (OMF). After trepanobiopsy, 20 out of 38 patients were confirmed as myelofibrotic (52.6\%), 5/38 (13.2\%) were diagnosed as ET, 1/38 (2.6\%) was diagnosed as myeloproliferative neoplasm (MPN), 6/38 (15.8\%) had polycythemia vera (PV). In six patients, the presence of disease was rejected. Patients with MF were divided into three groups according to the $J A K 2 \mathrm{~V} 617 \mathrm{~F}$ genotype status: homozygous for the mutation ( $3 / 20$ or $15.0 \%$ ), heterozygous $(9 / 20$ or $45.0 \%)$ and homozygous for the wild type allele $(8 / 20$ or $40.0 \%)$. The triggering factor of MF is still unknown. It was considered that this factor could have a genetic nature. Mutations in three genes were mainly accepted as an actual predisposing events to this disease: point mutations leading to amino acid substitutions in JAK2 (V617F) and in MPL (W515L, W515K), as well as inser-
\end{abstract}

\footnotetext{
${ }^{1}$ Department of Medical genetics, Medical Faculty, Medical University Sofia, Sofia, Bulgaria

${ }^{2}$ Clinic of Hematology and Oncology, University Hospital "St. Ivan Rilski," Sofia, Bulgaria
}

tion or deletion in CALR. We have proven that carriers of the $\mathrm{V} 617 \mathrm{~F}$ mutation prevailed in the group of patients with MF (altogether 12 patients or $60.0 \%$ ). Previous studies also showed that $J A K 2 \mathrm{~V} 617 \mathrm{~F}$ is present in more than half of MF patients within their blood-forming cells. Therefore, the risk of evolution to MF could be associated with V617Fmutant allele burden in patients with MPN.

Keywords: JAK2 (Janus kinase 2) V617F mutation; Myelofibrosis (MF); Myeloproliferative neoplasms (MPN).

\section{INTRODUCTION}

The existence of extramedullary hematopoiesis, overstimulated fibroblasts in abnormal microenvironment, fibrous tissue deposits in the bone marrow, dysregulation of the normal production of blood cells, low count of platelets and subsequently, the onset of anemia, are events typical for myelofibrosis (MF). Myelofibrosis could be Philadelphia chromosome positive or negative, primary or resulting as a consequence to initially diagnosed polycythemia vera (PV) or essential thrombocytopenia (ET) [1]. According to the diagnostic criteria of the World Health Organization (WHO), the V617F mutation on the Janus kinase 2 $(J A K 2)$ gene is listed among the major criteria for primary MF (PMF), ET and PV [1]. Mutations on three genes are associated with PMF: V617F (JAK2), ins/del (CALR), W515L/W515K (MPL) [2]. Different researchers have investigated the frequency of those genetic mutations in patients with ET, PMF and PV [3-8].

Janus kinase 2 exon 14 mutation in $J A K 2$ is the most frequent mutation in myeloproliferative neoplasms (MPN), present in the majority of PV and ET/PMF patients $(96.0$ and $65.0 \%$ in ET, respectively) [9]. It is considered to be the most significant potentiator of JAK/STAT signaling pathway leading to its constitutive activation [10]. Patients with ET positive for $J A K 2$ V617F slowly progress to PV [11], while PV and ET progress differently to second- 
ary MF [12]. The enhanced phosphorylation of STAT1 or STAT5 following JAK2 activation promotes megakaryopoiesis or erythropoiesis.

In the current study, we performed targeted mutational analysis of 232 patients from our Hematology Ward for $J A K 2$ V617F mutation. Thirty-eight (16.4\%) patients were suspected to carry PMF, and in 20, the diagnosis was confirmed by bone marrow biopsy. Our aim was to estimate the carriers of $J A K 2 \mathrm{~V} 617 \mathrm{~F}$ in our cohort of patients with $\mathrm{PMF}$ and compare it with the frequency so far reported by other researchers.

\section{MATERIALS AND METHODS}

Two hundred and thirty-two patients with different diagnoses (Figure 1) were referred for analysis to the genetic laboratory of the Clinic of Hematology, University Hospital "St.Ivan Rilski," Sofia, Bulgaria. DNA was extracted from venous blood of patients using a commercial kit (QIAamp DNA Blood Mini Kit; Qiagen Inc., Valencia, CA, USA). The procedure was executed in accordance with the manufacturer's recommendations. DNA samples were collected and analyzed for a total period of 3 years (from January 2016 to December 2018). All of the patients gave written informed consent for genetic analysis upon their acceptance at the Clinic.

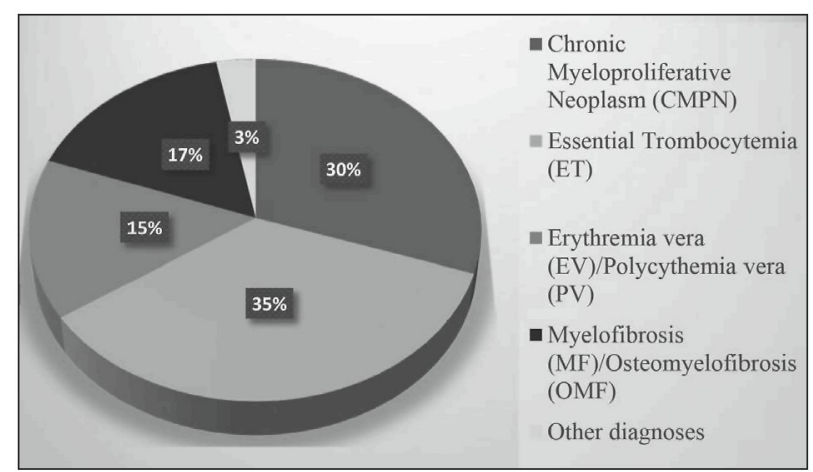

Figure 1. Diagnosis distribution of 232 Bulgarian patients. Other diagnoses (seven individuals) include: four patients with myelodysplastic syndrome (MDS), one with thrombocytosis, one with multiple myeloma and one with osteopetrosis.

JAK2 V617F Mutation Detection by Restriction Analysis. Previously isolated DNA samples were amplified using $J A K 2$ codon 617 mutation specific primers (V617F) (forward primer sequence: 5'-GGG TTT CCT CAG AAC GTT-3' and reverse primer sequence: 5'-TCA TTG CTT TCC TTT TTC-3'). Amplifications were performed for 32 cycles with Taq polymerase (Qiagen Inc.), annealing temperature of $60^{\circ} \mathrm{C}$, and standard amplification conditions. Polymerase chain reaction (PCR) of the human $J A K 2$ exon was performed as described by Baxter $e t$ al.
[13]. The amplified $460 \mathrm{bp}$ fragments were enzymatically digested using BsaXI restriction enzyme (New England Biolabs® Inc., Ipswich, MA, USA). Fragments of three different sizes $(241,189$ and $30 \mathrm{bp})$ resulted after digestion of the wild type allele, the mutant allele remained undigested (Figure 2). Digested fragments were separated in $2.0 \%$ agarose gel. Visualization of the restriction fragments was achieved by ethidium bromide.

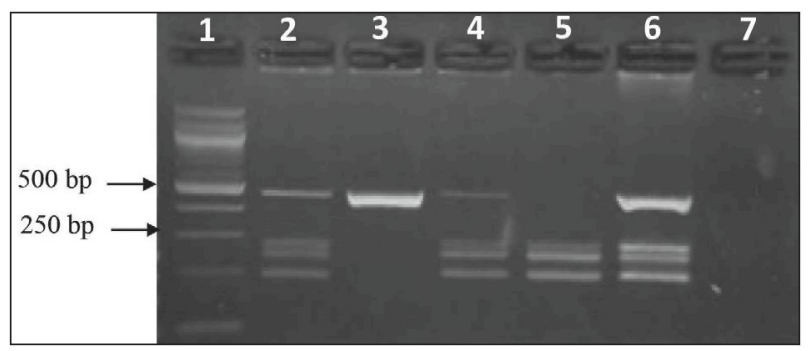

Figure 2. Restriction fragment length polymorphism analysis of the $J A K 2$ gene. BsaXI endonuclease was used to specifically cut the PCR product on 2.0\% agarose gel. Lane 1: 50 bp ladder; lane 3: sample from patient with two mutant alleles (homozygous by the mutant allele); lane 5: sample from patient with wild type alleles (homozygous by the wild type allele); lanes 2, 4 and 6: samples from patients with a mixed pattern of both mutant and wild type alleles.

\section{RESULTS}

Two hundred and thirty-two patients with chronic myeloproliferative neoplasms have been genotyped for their JAK2 V617F genotypic status. Eighty-two of 232 patients (35.4\%) were diagnosed with ET, 35/232 (15.1\%) PV/EV, 38/232 (16.4\%) MF/OMF (osteomyelofibrosis), $70 / 232(30.1 \%)$ chromic MPN and 7/232 (3.0\%) with other hematological diseases (Figure 1). We performed analysis of the bone marrow biopsy samples of patients with MF/ OMF (38 individuals) and in 20 of them (52.6\%) the initial diagnosis was confirmed. In six patients, the diagnosis was rejected based on the negative result of the biopsy sample (15.8\%), five patients eventually proved to carry ET (13.2\%), six had PV (15.8\%) and one had MPN (2.6\%).

The mean age of the patients in our study was 62 years (29-94 years), there was an equal distribution men:women (10 vs. 10) (Table 1). According to the $J A K 2$ genotype status: $8 / 20(40.0 \%)$ were homozygous with the wild type allele (NN) (men:women = 3:5); 9/20 (45.0\%) were heterozygous $(\mathrm{MN})$ (men:women = 6:3) and 3/20 (15.0\%) were homozygous with the mutated allele (MM) (men:women =1:2).

Overall, in 20 patients with $\mathrm{MF}$, proven by trepanobiopsy, $12(60.0 \%$ ) (group 1) carried the mutant allele and eight (40.0\%) (group 2) did not. The mean age of patients from group 1 was 61.4 years; men:women ratio was 7:5. Patients in group 2 showed similar characteristics (mean age 62.4 years; men:women ratio was $3: 5$ ). 
Table 1. Characteristics of patients with myelofibrosis (MF) confirmed by bone marrow biopsy. Below are shown parameters for sex, age and the genotype status according to the $J A K 2$ V617F mutation.

\begin{tabular}{|r|c|c|c|c|}
\hline \multicolumn{1}{|c|}{$\#$} & Year & Sex-Age & $\begin{array}{c}\text { Genotype Status } \\
\text { V617F Mutation }\end{array}$ & D $_{\mathbf{x}}$ \\
\hline 1 & 2015 & F-58 & NN & MF \\
\hline 2 & 2105 & M-65 & MN & MF \\
\hline 3 & 2016 & M-59 & MN & MF \\
\hline 4 & 2016 & M-60 & NN & MF \\
\hline 5 & 2016 & F-76 & MN & MF \\
\hline 6 & 2016 & F-29 & MN & MF \\
\hline 7 & 2017 & F-86 & NN & MF \\
\hline 8 & 2017 & M-51 & NN & MF \\
\hline 9 & 2017 & M-73 & MN & MF \\
\hline 10 & 2017 & M-54 & MN & MF \\
\hline 11 & 2017 & F-51 & NN & MF \\
\hline 12 & 2017 & M-94 & MM & MF \\
\hline 13 & 2017 & F-65 & MM & MF \\
\hline 14 & 2018 & F-76 & MM & MF \\
\hline 15 & 2018 & F-47 & NN & MF \\
\hline 16 & 2018 & M-63 & MN & MF \\
\hline 17 & 2018 & M-64 & NN & MF \\
\hline 18 & 2018 & F-87 & NN & MF \\
\hline 19 & 2018 & M-65 & MN & MF \\
\hline 20 & 2018 & F-56 & MN & MF \\
\hline
\end{tabular}

$\mathrm{D}_{\mathrm{x}}$ : diagnosis; $\mathrm{MF}$ : myelofribosis; $\mathrm{NN}$ : individuals homozygous for the normal wild type allele; $\mathrm{MN}$ : heterozygous; MM: individuals homozygous for the mutated allele.

\section{DISCUSSION}

Extramedullary hematopoiesis, overstimulated fibroblasts in abnormal microenvironment, fibrous tissue deposits in the bone marrow, dysregulation of the normal production of blood cells, low count of platelets and subsequently the onset of anemia are characteristic hallmarks of MF. Generally, it is a rare hematological event, predominantly diagnosed in elderly individuals aged over 50 years [14-16]. The MF/OMF patients were also rare in our dataset (20/232 patients confirmed the diagnosis, 8.6\%) and the mean age of the patients was 62 years. The gender does not affect the occurrence of the disease; there is an equal distribution men:women (10 vs. 10$)$.

The total survival time of patients with PMF can vary substantially, as well as the clinical course of the disease and its progression. It can be diagnosed as primary or developed as a part of other hematological malignancies as PV or ET. However, the mechanisms of development of $\mathrm{MF}$ are not completely understood. The $J A K 2$, together with CALR and MPL mutations, is a common triggering factor in Philadelphia chromosome-negative MPNs [17]. The so-called "driver" mutations activate the JAKSTAT signaling pathway. According to previous reports, about $85.0 \%$ of PMF patients have affected one of the three genes: $J A K 2(60.0-65.0 \%), M P L(5.0 \%)$ or $C A L R$ (20.0-25.0\%) [14]. In our study, 20 patients with MF were analyzed for $J A K 2$ mutation by quantitative PCR; and 12 of them carried the mutant allele (60.0\%), which is in accordance with the data reported by other researchers [4]. The remaining patients ( 12 individuals) who were accepted with an initial diagnosis of $\mathrm{MF}$, five were confirmed to carry ET, six with PV and one remained an unclassified MPN. This showed that trepanobiopsy is absolutely essential for the final precision of the diagnosis.

The discovery of the genetic transformation of the $J A K 2$ gene in the majority of our cases with MF makes possible their treatment with Jakafi (Ruloxitinib), which is an approved targeted drug for MF. It is indicated both for primary and secondary MF, either high- or intermediaterisk groups. It helps to reduce side effects such as splenomegaly, abdominal and bone pain. At the same time, it controls the blood levels of the inflammatory cytokines.

The $J A K 2$ V617F mutation leads to constitutive activation of JAK/STAT signaling [9]. As the $J A K 2$ mutation is present in only about $60.0 \%$ of patients with $\mathrm{MF}$, it could not be considered to be the only reason for this pathology. The triggering event of MF is not yet clarified and other mutations besides $J A K 2$ have recently been studied in relation to MF. They include mutations in genes such as $I D H 1, I D H 2, A S X L 1, S R S F 2$ and $E Z H 2$ [2]. In 2019, Wilms tumor 1 (WT1) expression was investigated in 152 patients with MPNs [18]. The progression of ET and PV to MF was accompanied by increased levels of $W T 1$ gene expression. Mutations in a tumor-suppressor gene, TP53, have also been detected in MPN, especially on older patients [19]. Some cytogenetic transformations (e.g., additional chromosome 8 , inversion of chromosome 3 and rearrangements of chromosome 11) have been considered unfavorable markers for MF [20].

Even though the number of MF patients in our study are quite few (20 individuals), we could underline the slight prevalence of males in the mutant allele carriers (seven men vs. five women), while in the group of non carriers, females prevailed slightly (three men $v s$. five women). As for the age of the patients, the registered difference between carriers and non carriers of the $J A K 2$ V617F mutation was 1 year (mean age of carriers:non carriers $=61.4: 62.4$ years). Whether the male gender and younger age are factors that contribute to $J A K 2$ mutational status is disputable and has to be further investigated in a larger cohort of MF patients. We have not had a case in which the $J A K 2$ mutation occurs simultaneously with other mutations, as it generally occurs as a single event. However, it could be associated with a different severity of symptoms from asymptomatic ET to MF [19]. We could speculate that other factors rather than genetic could modify the sever- 
ity of the disease (for example age, gender, comorbidity, microenvironment in the bone marrow, etc.) [19].

The frequency of MF in our cohort of patients (232 individuals) was estimated to be $8.6 \%$ (20 individuals). The distribution was equal between genders and the mean age (63.95 years) approaches the mean age given by other researchers (65 years). The majority of MF patients $(60.0 \%$ or 12 individuals) were $J A K 2$ V617F mutation-positive and thereby treatable by Jakafi (Ruxolitinib). For the other $40.0 \%$ of cases (eight individuals), treatment options were limited. They included erythropoiesis-stimulating agents, hydroxyurea and immunomodulatory drugs, which have unconvincing effect in improving patients' survival.

Declaration of Interest. The authors report no conflicts of interest. The authors alone are responsible for the content and writing of this article.

\section{REFERENCES}

1. Swerdlow SH, Campo E, Harris NL, Jaffe ES, Pileri $\mathrm{SA}$, Stein $\mathrm{H}$, et al. WHO Classification of Tumours of Haematopoietic and Lymphoid Tissues, 4th ed. Lyon, France: International Agency for Research on Cancer, 2008.

2. Rumi E, Pietra D, Pascutto C, Guglielmelli P, Martinez-Trillos A, Casetti I, et al. Clinical effect of driver mutations of JAK2, CALR, or MPL in primary myelofibrosis. Blood. 2014; 124(7): 1062-1069.

3. Klampfl T, Gisslinger H, Harutyunyan AS, Nivarthi H, Rumi E, Milosevic JD, et al. Somatic mutations of calreticulin in myeloproliferative neoplasms. N Engl J Med. 2013; 369(25): 2379-2390.

4. Cazzola M, Kralovics R. From Janus kinase 2 to calreticulin: The clinically relevant genomic landscape of myeloproliferative neoplasms. Blood. 2014; 123(24): 3714-3719.

5. Ha JS, Kim YK. Calreticulin exon 9 mutations in myeloproliferative neoplasms. Ann Lab Med. 2015; 35(1): 22-27.

6. Lavi N. Calreticulin mutations in myeloproliferative neoplasms. Rambam Maimonides Med J. 2014; 5(4): $\mathrm{e} 0035$.

7. Montoro J, Robledo C, Zamora L, Valcarcel D, Ramos F. Calreticulin mutations are exceedingly rare in patients with myelodysplastic syndromes with myelofibrosis. Ann Hematol. 2017; 96(2): 317-318.

8. Ebid GT, Ghareeb M, Salaheldin O, Kamel MM. Prevalence of the frequency of JAK2 (V617F) mutation in different myeloproliferative disorders in Egyptian patients. Int J Clin Exp Pathol. 2015; 8(9): 11555-11559.
9. Passamonti F, Maffioli M, Caramazza D, Cazzola M. Myeloproliferative neoplasms: From JAK2 mutations discovery to JAK2 inhibitor therapies. Oncotarget. 2011; 2(6): 485-490.

10. de Freitas RM, da Costa Maranduba CM. Myeloproliferative neoplasms and the JAK/STAT signaling pathway: An overview. Rev Bras Hematol Hemoter. 2015; 37(5): 348-353.

11. Campbell PJ, Scott LM, Buck G, Wheatley K, East CL, Marsden JT, et al. Definition of subtypes of essential thrombocythaemia and relation to polycythaemia vera based on JAK2 V617F mutation status: A prospective study. Lancet. 2005; 366(9501): 1945-1953.

12. Passamonti F, Rumi E, Pungolino E, Malabarba L, Bertazzoni $\mathrm{P}$, Valentini $\mathrm{M}$, et al. Life expectancy and prognostic factors for survival in patients with polycythemia vera and essential thrombocythemia. Am J Med. 2004; 117(10): 755-761.

13. Baxter EJ, Scott LM, Campbell PJ, East C, Fourouclas $\mathrm{N}$, Swanton S, et al. Acquired mutation of the tyrosine kinase JAK2 in human myeloproliferative disorders. Lancet. 2005; 365(9464): 1054-1061.

14. Lichtman M, Beutler E. Idiopathic myelofibrosis (agnogenic myeloid metaplasia). In: Beutler E, Editor. William's Hematology. New York, NY, USA: McGraw-Hill. 2001: 1125-1136.

15. Mavroudis D, Barrett B. Myelofibrosis (agnogenic myeloid metaplasia). In: Young N, Editor. Bone Marrow Failure Syndrome. Philadelphia, PA, USA: W.B. Saunders Company. 2000: 122-134.

16. Peterson P. Myelofibrosis. In: Kjeldsberg C, Editor. Myelofibrosis. Practical Diagnosis of Hematologic Disorders. Chicago, IL, USA: American Society for Clinical Pathology Press. 2010: 477-479.

17. Rego de Paula M Jr, Nonino A, Minuncio Nascimento $\mathrm{J}$, Bonadio RS, Pic-Taylor A, de Oliveira SF, et al. High frequency of copy-neutral loss of heterozygosity in patients with myelofibrosis. Cytogenet Genome Res. 2018; 154(2):62-70.

18. Cottin L, Riou J, Boyer F, Bouvier A, Zannetti A, Blouet A, et al. WT1 gene is overexpressed in myeloproliferative neoplasms, especially in myelofibrosis. Blood Cells Mol Dis. 2019; 75: 35-40.

19. O'Sullivan J, Mead AJ. Heterogeneity in myeloproliferative neoplasms: Causes and consequences. Adv Biol Regul. 2019; 71: 55-68.

20. Takenaka K, Shimoda K, Akashi K. Recent advances in the diagnosis and management of primary myelofibrosis. Korean J Intern Med. 2018; 33(4): 679-690. 